
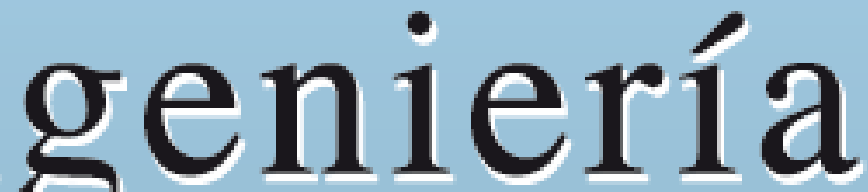

Revista de la Universidad de Costa Rica ENEROIDICIEMBRE 2002 - VOLUMEN 12 - $\mathrm{N}^{\circ} 1$ y 2

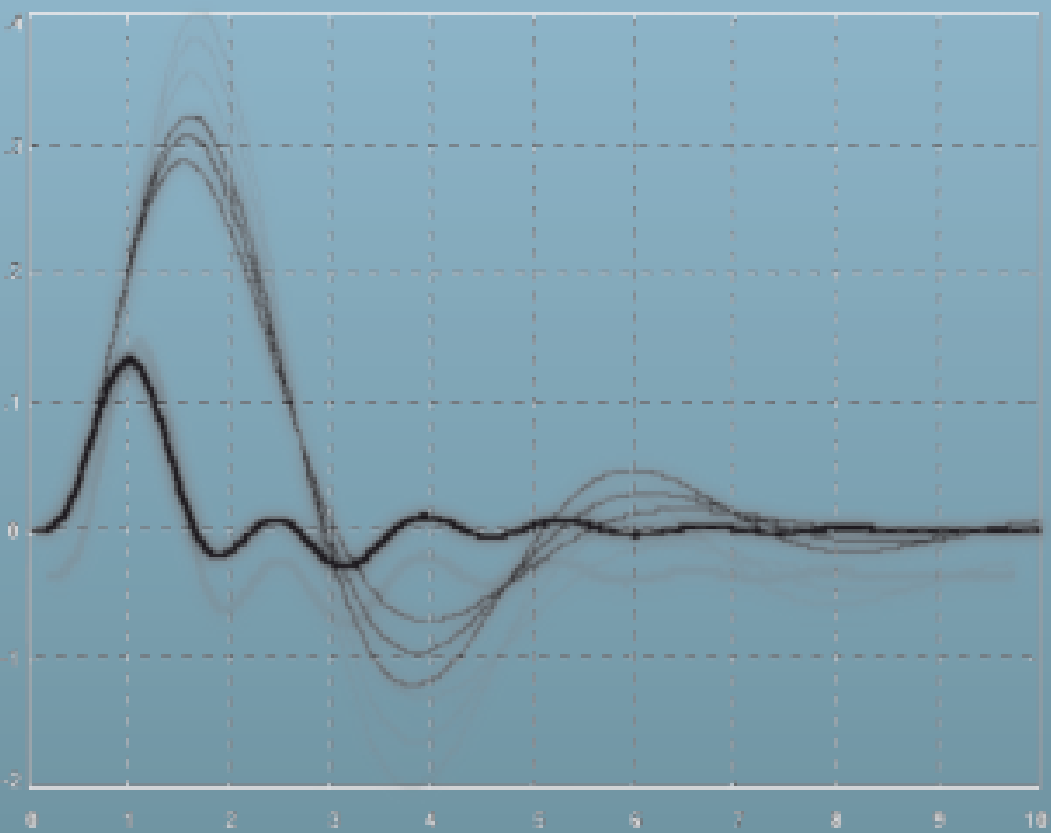




\title{
ECUACIONES PARA CONTROLADORES PID UNIVERSALES
}

Víctor M. Alfaro Ruíz.

\begin{abstract}
Resumen
Se propone la utilización de solamente dos ecuaciones para representar a los controladores PID.

El controlador PID Universal tipo Paralelo $\left(P I D_{u p}\right)$ y el controlador PID Universal tipo Serie $\left(P_{I} D_{u s}\right)$, junto con un conjunto único de parámetros que permiten simplificar y facilitar la utilización y el entendimiento de las características de los diferentes controladores PID disponibles en el mercado.
\end{abstract}

Palabras clave: controladores PID, ecuaciones universales

\begin{abstract}
Two general equations to describe the $P I D$ controllers are proposed. The Universal Parallel Type PID $\left(P I D_{u p}\right)$ and the Universal Serial Type PID $\left(P I D_{u s}\right)$, with a single set of parameters to simplified the use and allow a better understanding of the characteristics of the different commercial PID controllers.
\end{abstract}

Keywords: PID controllers, universal equations

\section{INTRODUCCIÓN}

En un lazo de control realimentado, como el mostrado en la Figura 1, las acciones de control son efectuadas por el instrumento denominado controlador, el cual contiene los modos o acciones de control que actúan sobre la señal de error $e(t)$. Este error es la diferencia entre el valor deseado de la variable controlada $r(t)$ y su valor real $y(t)$. Usualmente son características deseables del comportamiento del sistema de control, que lleve la variable controlada a un nuevo valor deseado en el caso de que este cambie, o que la regrese a su valor deseado ante la presencia de una perturbación $z(t)$, en el menor tiempo posible y con errores y oscilaciones mínimas. La forma en que el controlador realiza esto depende de su estructura y del valor de sus parámetros.

En diferentes publicaciones ${ }^{[7]}$ se ha reportado que el controlador PID (Proporcional - Integral Derivativo), en donde los términos proporcional, integral y derivativo se refieren a las acciones de control tomadas usualmente sobre el error, es el tipo de controlador más empleado en la industria, donde cerca del $95 \%$ de los lazos de control emplean un PID. Sin embargo y contrario a la creencia generalizada, no existe un algoritmo único para este controlador, ni tampoco una nomenclatura única para referirse a sus parámetros y características ${ }^{[10,11] \text {. }}$

El primer controlador que incorporó las tres acciones básicas de control anteriores, fue el Fulscope modelo 100 introducido al mercado por Taylor Instruments en $1940^{[3]}$. Las diferencias entre la forma en que este controlador fue realizado y la ecuación generalmente utilizada para la descripción de las acciones de un controlador PID originaron, desde la creación de éste, múltiples y diversas formas de referirse a él ${ }^{[12,13]}$.

Se encuentran en la literatura técnica nombres tales como controlador PID, ideal, serie, industrial, paralelo, PI-D, I-PD, de un grado de libertad, de 
dos grados de libertad, interactuante, no interactuante, clásico, de ganancias independientes, estándar, ISA, tipo $A, B, C$, o $D$, y mezclas de éstos para referirse a este tipo de controlador. Multiplicidad de términos que solo contribuyen a la confusión sobre la forma en que sus acciones están relacionadas, ya que incluso se puede encontrar que se utilizan los mismos nombres para denominar formas diferentes. deseadas que un controlador PID moderno debe poseer, pero no establecen ecuaciones generales que permitan su normalización.

Se considera entonces, no solo conveniente si no necesario, contar con el mínimo de ecuaciones posibles que permitan describir el funcionamiento de las diferentes variantes de los controladores $P I D$ disponibles en el mercado.



Figura 1. Sistema de control realimentado

Por ejemplo, la información sobre el programa de análisis de lazos de control y sintonización de controladores ExperTune ${ }^{[8]}$, indica que este posee una base de datos de más de 200 controladores industriales de diferentes fabricantes. Por su parte, el manual de aplicaciones del programa de optimización de sistemas de control PROTUNER $32^{[15]}$ incluye una tabla comparativa de 75 controladores PID, los que evidentemente no son todos diferentes entre sí, pero da una muestra clara de que no existen algoritmos estandarizados.

En cuanto a la denominación de los parámetros de cada acción de control, también se encuentra variedad de términos y unidades diferentes según sean las preferencias de sus respectivos fabricantes.

EnTech $^{[6]}$ por un lado y Gerry y Shinskey ${ }^{[10]}$ por otro, enumeran algunas de las características

\section{CONTROLADORES}

Las funciones de transferencia de los controladores PID son alguna variante de las siguientes estructuras básicas ${ }^{[1,11]}$ :

- Controlador PID-Ideal

$u(s)=K_{C}\left[1+\frac{1}{T_{i} s}+\frac{T_{d} s}{\tau_{f d} s+1}\right](r(s)-y(s))$

- Controlador PID-Serie

$u(s)=K_{C}^{\prime}\left[1+\frac{1}{T_{i}^{\prime} s}\right]\left[1+\frac{T_{d}^{\prime} s}{\tau_{f d} s+1}\right](r(s)-y(s))$

cuyos diagramas de bloques se muestran en las Figuras 2 y 3 y en los cuales se puede apreciar que mientras en el controlador ideal las tres acciones de 
control están en paralelo, en el otro estas están una detrás de la otra, lo cual corresponde a poner un controlador $P I$ en serie con un $P D$. Esta diferencia en la forma de construir los controladores PID se originó precisamente con la fabricación del Fulscope modelo 100, ya que la fabricación de un controlador serie requiere menos amplificadores que uno en paralelo, reduciéndose así su costo.
La mayoría de los controladores neumáticos y electrónicos analógicos fabricados son tipo serie $^{[4]}$, mientras que en los actuales controladores electrónicos digitales, es usualmente posible seleccionar el tipo de ecuación del PID deseado, aunque no todos los fabricantes brindan información completa de cómo están construidos internamente sus controladores.

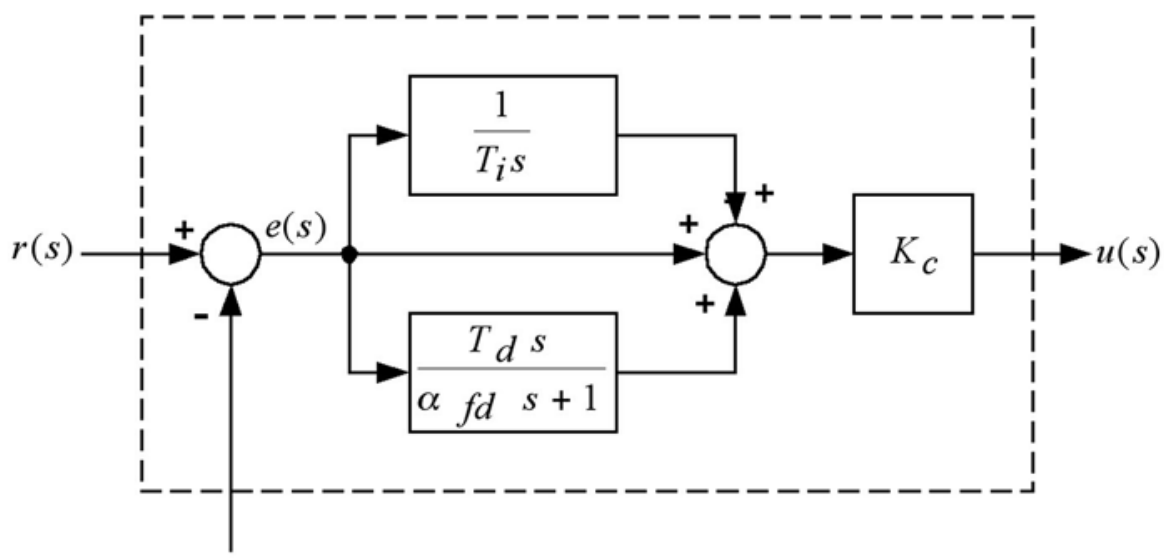

$y(s)$

Figura 2. Controlador PID Ideal

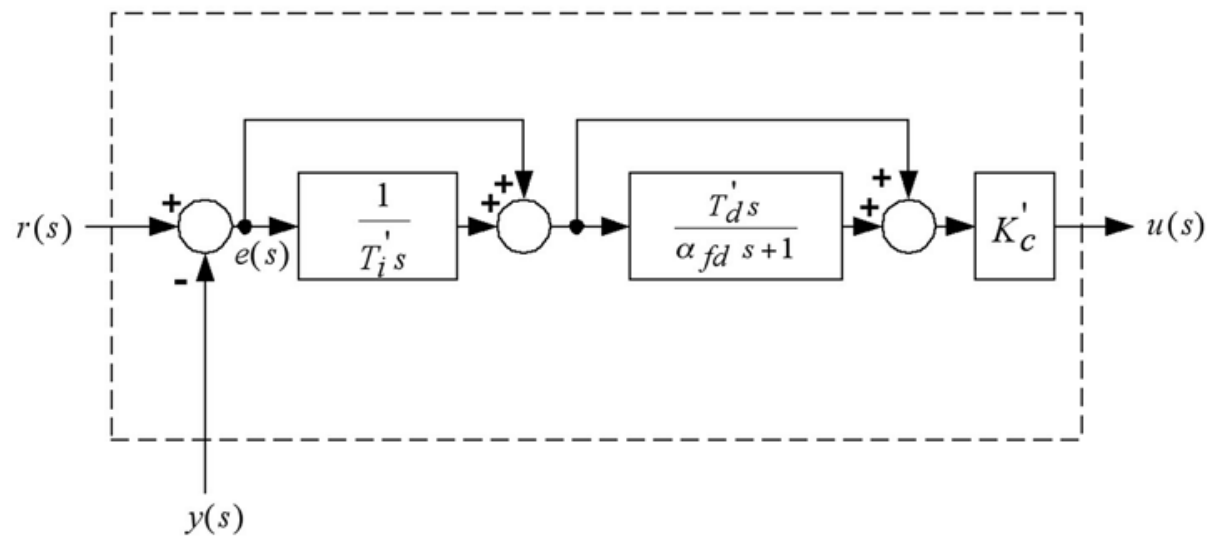

Figura 3. Controlador PID Serie 


\section{ECUACIONES PARA CONTROLADORES PID UNIVERSALES}

Como no es posible describir por medio de una sola ecuación la estructura paralela y la serie, se proponen, entonces, dos ecuaciones generales o universales para representar los controladores $P I D$, una basada en una estructura paralela y otra en una serie.

Un controlador PID Universal tipo Paralelo $\left(\right.$ PID $\left._{\text {up }}\right)$ será aquel en que las acciones de control integral y derivativo no interactúan una con la otra, como se muestra en la Figura 4, y estará representado por la ecuación (3).

Un controlador PID Universal tipo Serie (PID $\left.{ }_{\mathrm{us}}\right)$ será aquel que resulta de la colocación en serie de las acciones integral y derivativa, como se muestra en la Figura 5, y estará representado por la ecuación (4).

En las ecuaciones (3) a (4) las variables y parámetros son:

$r(t) \quad$ valor deseado

$u_{p}(t) \quad$ salida del controlador tipo paralelo

$u s(t) \quad$ salida del controlador tipo serie

$y(t) \quad$ variable controlada

$K_{c p} \quad$ ganancia, controlador paralelo

$T_{i p} \quad$ tiempo integral, controlador paralelo

$T_{d p} \quad$ tiempo derivativo, controlador paralelo

$K_{c s} \quad$ ajuste proporcional, controlador serie

$T_{i s} \quad$ ajuste integral, controlador serie

$T_{d s} \quad$ ajuste derivativo, controlador serie

$K_{d} \quad$ selector, acción derivativa $\left(\mathrm{K}_{\mathrm{d}}=0\right.$ o 1$)$

$K_{r} \quad$ factor de peso del valor deseado $\left(0 \leq K_{r} \unlhd\right)$ $\alpha_{d} \quad$ constante del filtro derivativo. $\left(0,05 \leq \alpha_{d} \leq 0,20\right)$, normalmente, es fijado por el fabricante en 0.1

$\tau_{\mathrm{f}} \quad$ constante de tiempo, filtro PID-IMC

$\tau_{r} \quad$ constante de tiempo, filtro del valor deseado

$p \quad$ operador derivada $(d / d t)$

\section{CARACTERÍSTICAS DE LOS PID UNIVERSALES}

Las ecuaciones propuestas para los controladores $P I D$ universales tipo Serie $P I D_{u s}$ y tipo Paralelo $P I D_{u p}$, con excepción de la forma en que el modo integral y derivativo están dispuestos, presentan un conjunto de parámetros de sintonización y características comunes. Estas permiten representar una gran variedad de controladores PID disponibles en el mercado y unificar la forma en que se hace referencia a sus características y procedimientos de sintonización.

Se conservan los parámetros básicos de sintonización de los modos de control proporcional, integral y derivativo, como la ganancia del controlador $\left(K_{c s}, K_{c p}\right)$, la constante de tiempo del modo integral o simplemente tiempo integral $\left(T_{i s}, T_{i p}\right) \mathrm{y}$ la constante de tiempo del modo derivativo o tiempo derivativo $\left(T_{d s}, T_{d p}\right)$.

\subsection{Aplicación del modo derivativo}

El modo derivativo está representado por un derivador real, incluye un filtro paso bajo en el que la constante ad permite seleccionar la posición del polo. Esta constante generalmente es fija, aunque algunos fabricantes permiten su selección en un

$$
\begin{gathered}
u_{p}(t)=K_{c p}\left[\frac{K_{r}}{\tau_{r} p+1} r(t)-y(t)+\frac{1}{T_{i p} p}(r(t)-y(t))+\frac{T_{d p} p}{\alpha_{d} T_{d p} p+1}\left(K_{d} r(t)-y(t)\right)\right]\left(\frac{1}{\tau_{f} p+1}\right) \\
u_{S}(t)=K_{C S}\left[\left(\frac{K_{r}}{\tau_{r} p+1}+\frac{1}{T_{i s} p}\right)\left(1+\frac{K_{d} T_{d s} p}{\alpha_{d} T_{d s} p+1}\right) r(t)-\left(1+\frac{1}{T_{i s} p}\right)\left(1+\frac{T_{d s} p}{\alpha_{d} T_{d s} p+1}\right) y(t)\right]\left(\frac{1}{\tau_{f} p+1}\right)
\end{gathered}
$$


ámbito de valores bastante restringido. Como regla general el polo del derivador se encuentra aproximadamente diez veces más a la izquierda que el cero, por lo que su influencia en la respuesta del controlador es despreciable, no siendo necesario incluirlo en los estudios analíticos de los lazos de control.

Una de las características que origina diferentes ecuaciones y nombres para los controladores PID es la forma en que el modo derivativo es utilizado, si éste se aplica a la señal de error o solamente a la señal realimentada.

Aunque tradicionalmente se entiende que los tres modos de control actúan sobre la señal de error para lograr su eliminación, en el caso de cambios bruscos y frecuentes del valor deseado, el modo derivativo introducirá saltos indeseables en la señal de salida del controlador, por lo que es frecuente que éste se aplique solamente a la señal realimentada.

Es importante hacer notar que excepto en los instantes en que el valor deseado cambia, la derivada del error y la derivada del negativo de la señal realimentada es idéntica; de esta manera, la selección de la señal que actúa sobre el modo derivativo no tiene ninguna incidencia sobre el comportamiento de los lazos de control cuando su funcionamiento es como regulador, aplicación dominante en la industria.

El selector de la acción derivativa $K_{d}$ permite establecer la forma en que se aplica el modo derivativo, si es 1,0 actúa sobre el error y si es 0,0 actúa solo sobre la señal realimentada.

\subsection{Peso y filtrado del valor deseado}

Como se muestra en la Figura 1 un lazo de control tiene dos entradas, el valor deseado $r(t)$ y la perturbación $z(t)$, esto obliga a sintonizar el controlador teniendo en cuenta el funcionamiento deseado del lazo de control, ya sea como servomecanismo para los casos en que el valor deseado cambia en forma continua y se requiere un buen seguimiento del mismo o como regulador en los casos en que el valor deseado permanece constante y se necesita insensibilidad a las perturbaciones. La práctica ha demostrado que un lazo de control sintonizado como servomecanismo no funciona en la mejor forma como regular y que el desempeño de uno sintonizado como regulador a su vez no es satisfactorio como servomecanismo. En este último caso la respuesta del sistema de control a un cambio en el valor deseado es más oscilatoria y suele presentar un sobrepaso máximo excesivo.

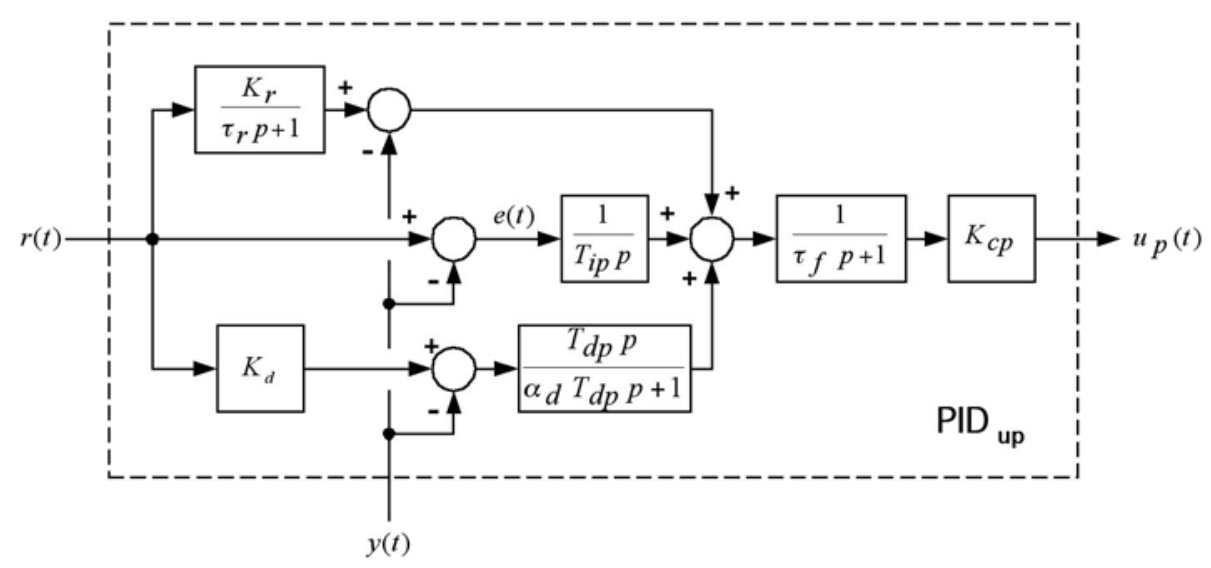

Figura 4. Controlador PID Universal tipo Paralelo 


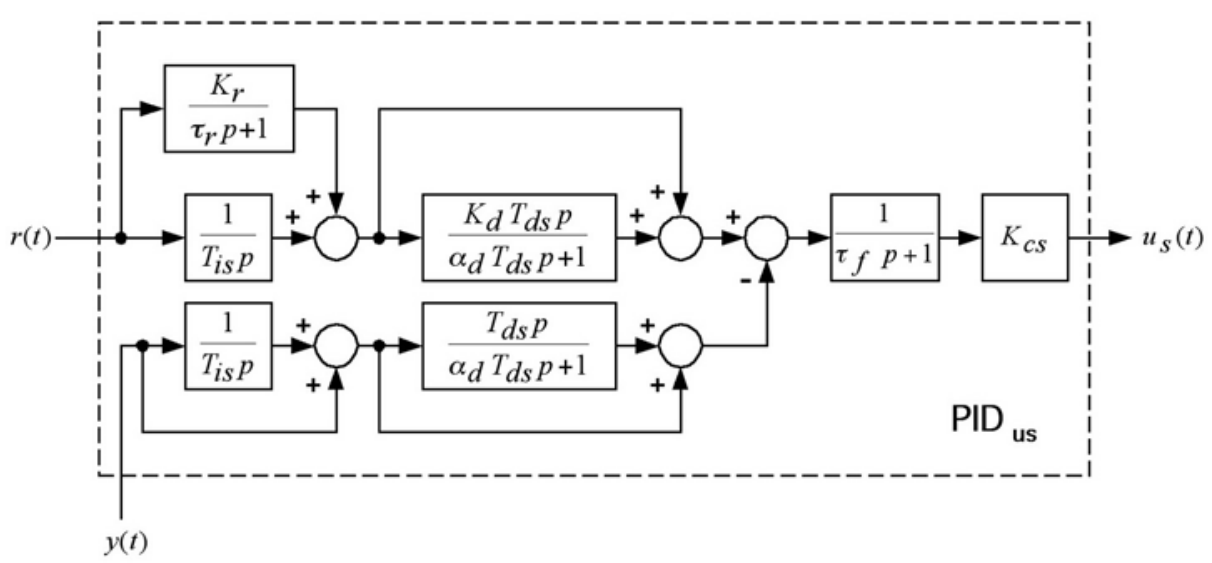

Figura 5. Controlador PID Universal tipo Serie

Hemos señalado que el funcionamiento más frecuente de los lazos de control en la industria es como regulador con cambios poco frecuentes del valor deseado. Como no se puede lograr un comportamiento adecuado del lazo en respuesta a los cambios en el valor deseado en forma simultanea con su funcionamiento óptimo como regulador, algunos fabricantes proveen la opción de que el modo proporcional actúe solo sobre la señal realimentada y no sobre el error.

En adición, en los últimos años los fabricantes han incorporado un factor de peso o incluso una constante de tiempo para un filtro del valor deseado, lo que agrega un parámetro de sintonización, creándose los denominados controladores de dos grados de libertad. Con este tipo de controlador se pueden seleccionar sus parámetros tradicionales $\left(K_{c}, T_{i}, T_{d}\right)$ para lograr un funcionamiento óptimo como regulador y utilizar el factor de peso y las características de filtrado del valor deseado para mejorar su desempeño como servomecanismo.

En las ecuaciones universales propuestas, las características de estos nuevos controladores están incorporadas mediante un factor de peso del valor deseado $K_{r}$, que puede variarse ente 0,0 y 1,0 y una constante de tiempo $\tau_{\mathrm{r}}$ del filtro para este.

\subsection{Filtro para controladores PID-IMC}

Además de las técnicas tradicionalmente empíricas para la selección de los parámetros de sintonización de los controladores PID, en los últimos años se han desarrollado técnicas de sintonización analíticas para estos controladores a partir de la teoría de Control con Modelo Interno o $I M C^{[9]}$ por sus siglas en inglés, conduciendo a lo que denominaremos controladores PID-IMC.

Estas técnicas han cobrado popularidad en las aplicaciones industriales para la sintonización de lazos de control que operan como servomecanismos. Ligan los parámetros del controlador a un solo parámetro de diseño, usualmente denominado $\lambda$, el cual determina el comportamiento dinámico y la robustez del lazo de control. Las ecuaciones desarrolladas para el cálculo de los parámetros de los controladores por Rivera et al. ${ }^{[14]}$, Brosilow $^{[5]}$ y otros autores, conducen en ocasiones a un controlador PID en serie con un retazo o filtro de primer orden.

Los controladores más modernos, usualmente incorporan este filtro adicional requerido en la salida. En las ecuaciones universales propuestas, la constante de tiempo $\tau_{f}$ permite la sintonización del mismo. 


\subsection{Características adicionales de los controladores PID comerciales}

Además de las características descritas anteriormente sobre la aplicación de los modos de control, los controladores comerciales deben incluir otras funciones. Se puede contar con un selector de acción directa o inversa, transferencia sin saltos del modo manual al automático, variación de la salida del controlador en modo manual, prevención de la saturación del modo integral o anti-reset windup, como se le denomina en inglés, realimentación externa y modo local o remoto para el valor deseado.

También podrían incluir un filtro de la señal de entrada para la reducción del ruido de medición, una lista de ganancias o tabla de conjuntos de parámetros para el control de plantas no lineales y opciones para control en adelanto o pre-alimentado. Posiblemente, dependiendo del fabricante hay otras características optativas que en su mayoría no están relacionadas directamente con la selección de los parámetros, razón por la cual no se incluyen dentro de las ecuaciones universales propuestas.

\subsection{Conversión de parámetros}

Debido a la existencia de dos posibles formas generales para desarrollar un controlador PID Universal, serie o paralela, es necesario establecer ecuaciones de conversión entre sus parámetros.

Eliminando las constantes de tiempo de los filtros y tomando los valores de las constantes de las opciones como unitarios, las ecuaciones (3) y (4) básicas se reducen respectivamente a las formas:

$$
\begin{aligned}
& u_{p}(t)=K_{c p}\left[1+\frac{1}{T_{i p} p}+T_{d p} p\right] e(t) \\
& u_{S}(t)=K_{C S}\left[1+\frac{1}{T_{i s} p}\right]\left[1+T_{d s} p\right](t)
\end{aligned}
$$

Lo cual nos permite determinar las ecuaciones de conversión de los parámetros.

\subsubsection{Tipo paralelo a serie}

Los parámetros de un controlador PID Universal tipo Serie equivalente a uno tipo Paralelo son:

$$
\begin{aligned}
K_{c s} & =K_{c p} K_{p s} \\
T_{i s} & =T_{i p} K_{p s} \\
T_{d s} & =T_{d p} / K_{p s}
\end{aligned}
$$

donde

$$
K_{p s}=0.5\left(1+\sqrt{1-4 T_{d p} / T_{i p}}\right)
$$

es el factor de conversión del PID Universal Paralelo al PID Universal Serie.

Como se puede ver en (8) no siempre existirá un controlador serie equivalente a uno paralelo dado. Se requiere que

$$
T_{i p} \geq 4 T_{d p}
$$

Los dos ceros del controlador serie son siempre reales mientras que los del controlador paralelo pueden ser reales o complejos conjugados.

\subsubsection{Tipo serie a paralelo}

Los parámetros de un controlador PID Universal tipo paralelo equivalente a uno tipo serie son:

$$
\begin{aligned}
& K_{c p}=K_{s c} K_{s p} \\
& T_{i p}=T_{i s} K_{s p} \\
& T_{d p}=T_{d s} / K_{s p} \\
& \text { donde } \quad K_{s p}=1+T_{d s} / T_{i s}
\end{aligned}
$$

es el factor de conversión del PID Universal Serie al PID Paralelo. 
Como se puede ver en (11) siempre existirá un controlador paralelo equivalente a uno serie dado, razón por la que se considera más general al controlador PID tipo paralelo.

\section{REPRESENTACIÓN DE LOS PID COMERCIALES}

La mejor utilidad de las ecuaciones universales que se proponen para los controladores no radica en el estudio analítico o en la simulación de los lazos de control, sino en que sean utilizadas por los fabricantes para la estandarización de la nomenclatura relacionada con los controladores PID. Esto eliminaría la confusión y problemas que tienen los usuarios en el momento de poner en funcionamiento un lazo de control, máxime si se cambia un controlador por el de otro fabricante.

En el Apéndice se muestran las ecuaciones de los controladores dadas por tres fabricantes distintos. Por ejemplo, la Ecuación B - interactuante de Honeywell, el PID estándar de Moore y el PID clásico de Bailey-, parecen ser diferentes, aunque en la realidad corresponden al mismo tipo de controlador.

Las tres ecuaciones proporcionadas por Honeywell para sus controladores interactuantes se pueden reducir a una tabla de valores de los parámetros fijos como la siguiente:

\section{Controlador Honeywell Interactuante} Ecuación Universal tipo Serie PID ${ }_{u s}$

$\begin{array}{ccccccccc} & \boldsymbol{K}_{\boldsymbol{c s}} & \boldsymbol{T}_{\boldsymbol{i s}} & \boldsymbol{T}_{\boldsymbol{d} \boldsymbol{s}} & \boldsymbol{K}_{\boldsymbol{r}} & \tau_{\boldsymbol{r}} & \alpha_{\boldsymbol{d}} & \boldsymbol{K}_{\boldsymbol{d}} & \tau_{\boldsymbol{F}} \\ \mathrm{A} & \mathrm{Kc} & \mathrm{Ti} & \mathrm{Td} & 1 & 0 & 0,1 & 1 & 0 \\ \text { B } & \mathrm{Kc} & \mathrm{Ti} & \mathrm{Td} & 1 & 0 & 0,1 & 0 & 0 \\ \mathbf{C} & \mathrm{Kc} & \mathrm{Ti} & \mathrm{Td} & 0 & 0 & 0,1 & 0 & 0\end{array}$

\section{CONCLUSIONES}

Se propone la utilización de solamente dos ecuaciones para representar la gran variedad de controladores PID fabricados como una forma de simplificar el entendimiento de sus diferentes características, y facilitar el proceso de sintonización de los mismos en instalaciones que podrían utilizar controladores de una gran variedad de fabricantes.

La ecuación del controlador PID Universal tipo Paralelo $\left(P I D_{u p}\right)$ y la del controlador PID Universal tipo Serie $\left(P I D_{u s}\right)$, permiten reducir la descripción de las características particulares de un controlador al proporcionar una tabla con los valores, ya sean fijos o variables, permitidos para los diferentes parámetros que aparecen en éstas.

El eventual establecimiento de ecuaciones estándar (iguales o variantes de las propuestas por organizaciones como ISA The Instrumentation, Systems and Automation Society), contribuirá a que tras sesenta años de existencia se logre adecuar la terminología utilizada en los controladores PID, al cual le restan todavía muchos años de servicio eficiente ${ }^{2}$.

\section{APÉNDICE}

A continuación algunas ecuaciones de controladores PID tal como las muestran los fabricantes en su literatura.

\section{A.1 Honeywell ${ }^{1}$}

Ecuación A - Interactuante

$C V_{S}=K^{*}\left[\frac{1+T 1^{*} s}{T 1^{*} s} * \frac{1+T 2^{*} s}{1+a^{*} T 2^{*} s} *(P V P s-S P P s)\right]$

Ecuación B - Interactuante

$C V_{S}=K^{*}\left[\frac{1+T 1^{*} s}{T 1^{*} s} * \frac{1+T 2 * s}{1+a^{*} T 2 * s} * P V P s-\frac{1}{T 1^{*} s} * S P P s\right]$

Ecuación C - Interactuante

$C V_{S}=K^{*}\left[\left(\frac{1+T 1^{*} s}{T 1^{*} s}+\frac{T 2 * s}{1+a^{*} T 2 * s}\right) *(P V P s-S P P s)\right]$

Ecuación A - No Interactuante.

$C V_{S}=K^{*}\left[\left(\frac{1+T 1^{*} s}{T 1^{*} s}+\frac{T 2 * s}{1+a^{*} T 2 * s}\right) * P V P s-\frac{1+T 1 * s}{T 1^{*} s} S P P s\right.$ 
Ecuación B - No Interactuante

$C V_{S}=K^{*}\left[\left(\frac{1+T 1^{*} s}{T 1^{*} s}+\frac{T 2 * s}{1+a^{*} T 2 * s}\right) * P V P s-\frac{T 1^{*} s}{T 1^{*} s} S P P s\right.$

Ecuación $\mathrm{C}$ - No Interactuante

$C V_{S}=K *\left[\frac{1+T 1 * s}{T 1 * s} * \frac{1+T 2 * s}{1+a * T 2 * s} * P V P s-\frac{1+T 1 * s}{T 1 * s} * S P P s\right.$

$T 1$ - tiempo integral, $T 2$ - tiempo derivativo, constante $a=1 / 10$ interactuante, $a=1 / 16$ no interactuante.

\section{A2. Moore Products ${ }^{2}$}

Algoritmo PID Estándar

$\operatorname{OUT}(s)= \pm P G\left[\left(1+\frac{\tau D^{s}}{\left(\frac{\tau D}{D G}\right) s+1}\right) P V(s)-S P(s)\right]\left[1+\frac{1}{\tau^{I} s}-\mathrm{P}\right.$

$\mathrm{G}$ - ganancia proporcional, $\tau_{I}-$ constante de tiempo integral, $\mathrm{t}_{D}$ - constante de tiempo derivativa, $D G$ - ganancia derivativa.

\section{A3. Bailey Controls ${ }^{3}$}

Controlador PID Clásico

OUTPUT $=K K P\left(1+\frac{K_{I} / 60}{s}\right)\left[S P-\left(\frac{60 K D^{s+1}}{\frac{60 K D}{K} s+1}\right) P V\right.$

Controlador PID no interactuante

OUTPUT $=K\left(K P+\frac{K_{I} / 60}{s}\right)$ error $-K\left(\frac{60 K D^{s}}{\frac{60 K D}{K_{A}} s+1}\right) P V$

\section{NOTAS}

${ }^{1}$ Honeywell TDC 3000 Manual, 8.14 Control Algorithms

${ }^{2}$ Evolution of The PID Algorithm at Moore Products Co.

${ }^{3}$ Bailey Function Code 156 - Advanced PID Controllers

\section{BIBLIOGRAFÍA}

1. Alfaro, V.M..; “ ¿Son todos los controlado res PID iguales?, Ingeniería (Costa Rica), Vol. $3 \mathrm{~N}^{\mathrm{o}}$ 1, 1993.

2. Aström, K.J. y T. Hägglund, "The Futureof PID Control", IFAC Workshop on Digital Control: Past, Present and Future of PID Control, Terrasa, España, Abril 5 - 7, 2000.

3. Babb, M.; "Pneumatic Instruments Gave Birth to Automatic Control", Control En-gi neering (EUA), Vol. 37 Nº 12 (2), Oct. 1990.

4. Bennett, S.; "The Past of PID Controllers", IFAC Workshop on Digital Control: Past, Present and Future of PID Control, Te rrasa, España, Abril 5 - 7, 2000.

5. Brosilow, C.B.; "PI/PID Controller Para meters From IMC Designs", Department of Chemical Engineering, Case West Re-serve University, 1992.

6. EnTech Control Engineering ; "Automatic Controller Dynamic Specification", Re-su men de la Versión 1.0, Nov. 1993.

7. Erbay, A.S.; "An Overview on PID Control", RTP Corp., 2000.

8. ExperTune Inc.; "What ExperTune Needs to Know About Your PID Algorithm", ExperTune Inc., 2000.

9. Garcia, C.E. y M. Morari; "Internal model Control 1. A Unified Review and Some New Results", Ind. Eng. Chem. Process Des. Dev. (EUA), Vol. 21 N$^{\circ}$ 2, pág. 308 - 332, 1982.

10. Gerry, J. y F.G. Shinskey; "PID Controller Specification (White Paper)", Exper-Tune Inc., 2000.

11. Gerry, J. ; "A Comparison of PID Control Algorithms", Control Engineering (EUA), Vol 34 N N $^{\circ}$, Marzo 1987. 
12. Lipták, B. ; "Controller Tuning I: Concepts and Definitions", Control Engineer-ing On Line, Feb. 2001.

13. Lipták, B. ; "Controller Tuning II: Problems and Methods", Control Engineering On Li ne, Feb. 2001.

14. Rivera, D.E., M. Morari y S. Skogestad; "Internal model Control 4. PID Controller Design", Ind. Eng. Chem. Process Des. Dev. (EUA), Vol. 25, pág. 252 - 285, 1986.

15. Techmation, Inc.; "PROTUNER 32 Ap-pli cation Manual", Enero 1999.

\section{SOBRE EL AUTOR}

\section{Víctor M. Alfaro}

Profesor Asociado, Departamento de Automática, Escuela de Ingeniería Eléctrica, Universidad de Costa Rica

Apartado postal 2-10, 2060 UCR, San José, Costa Rica

Tel. 207-4472, Fax. 207-4139, correo electrónico: valfaro@eie.ucr.ac.cr

El presente trabajo fue realizado como parte del proyecto $N^{o} 731-A 0-169$ inscrito en la Vicerrectoría de Investigación de la Universidad de Costa Rica. 